\title{
Funcionalização do Polipropileno com Viniltrietoxisilano em Solução e no Estado Fundido.
}

\author{
Sônia M. B. Nachtigall, Afonso H. O. Felix, Telmo Ojeda e Raquel S. Mauler
}

\begin{abstract}
Resumo: Polipropileno (PP) foi funcionalizado com viniltrietoxisilano (VTES) em solução e no estado fundido, utilizando peróxidos como iniciadores. A influência da concentração de silano e de peróxido sobre o grau de funcionalização foi avaliada por Espectrometria no Infravermelho com Transformada de Fourier (FTIR). As reações feitas no estado fundido resultaram em produtos com maior grau de funcionalização. Medidas de torque e determinações de peso molecular por Cromatografia de Permeação em Gel (GPC) demonstraram a ocorrência de reações de quebra de cadeia em todos os sistemas, porém mais acentuadamente nas reações feitas no estado fundido.
\end{abstract}

Palavras-chave: Funcionalização de polipropileno, vinilsilano, solução, estado fundido, peso molecular.

\section{Introdução}

O polipropileno (PP) foi o polímero que apresentou o maior crescimento em aplicações comerciais na última década e, provavelmente, esta situação deve se manter durante algum tempo ${ }^{[1]}$. Baixo custo, performances similares às de muitos materiais de engenharia, facilidade de processamento e reciclagem são algumas das razões que fazem com que o PP ocupe uma posição de destaque entre os polímeros sintéticos. Entretanto, suas aplicações são limitadas devido a sua baixa polaridade e ausência de grupos funcionais ${ }^{[2]}$. A modificação química do PP por meio da introdução de grupos polares possibilita a obtenção de novas propriedades que podem levar à expansão do mercado de aplicações sem afetar significativamente a cadeia polimérica.

A funcionalização do PP tem sido feita com sucesso por meio de reações em solução ${ }^{[3-6]}$, no estado fundido $^{[7-9]}$ e no estado sólido ${ }^{[10-13]}$. Em geral, as rea- ções são feitas utilizando-se peróxidos, que geram radicais livres na cadeia polimérica, aos quais se adicionam moléculas polare ${ }^{[14]}$, tais como: fumarato de $\operatorname{dietila}^{[5]}$, anidrido maleico ${ }^{[9,15]}$, acetato de vinila ${ }^{[16]}$, ácido acrílico ${ }^{[17,18]}$, maleato de dietila ${ }^{[19,20]}$, anidrido itacônico $^{[21]}$ e isocianatos ${ }^{[22]}$.

As primeiras etapas do mecanismo de incorporação de monômeros vinílicos ao PP são normalmente descritas na literatura como segue ${ }^{[23]}$ : A quebra homolítica de cada molécula de peróxido orgânico produz dois radicais (Esquema 1), sendo que a velocidade de decomposição do peróxido depende somente da temperatura. Os radicais formados abstraem um átomo de hidrogênio da cadeia polimérica, produzindo macro-radicais. Devido à maior estabilidade dos radicais terciários, são abstraídos preferencialmente átomos de hidrogênio ligados a carbonos terciários no PP. Para as etapas seguintes, existem duas possibilidades diferentes: os 


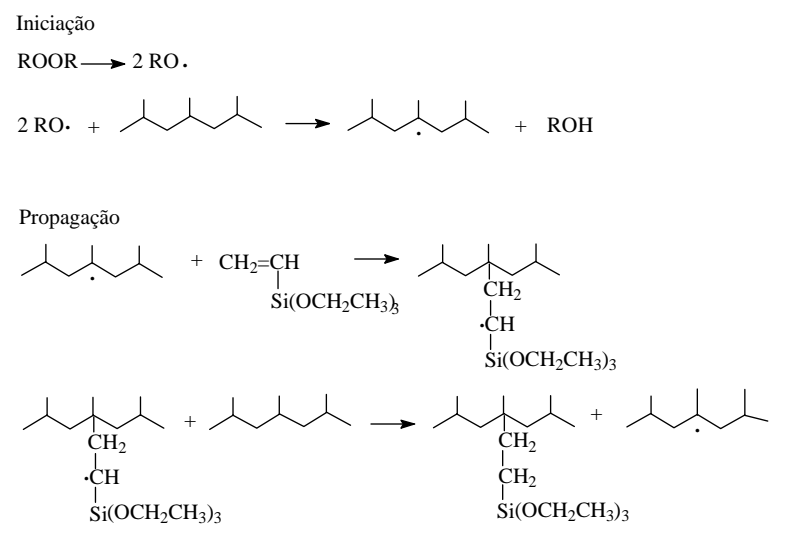

Reações de quebra de cadeia

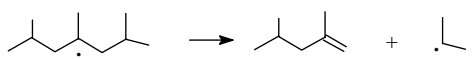

Esquema 1.

macro-radicais formados podem adicionar-se à molécula do monômero vinílico ou podem sofrer reações de cisão- $\beta$, produzindo cadeias de menor peso molecular ${ }^{[13]}$.

Nos últimos anos o uso de silanos na funcionalização de poliolefinas tem sido citado em inúmeras patentes $^{[24-29]}$. Entretanto, na literatura científica poucos trabalhos são encontrados, sendo que a maior parte dos mesmos visa à obtenção de poliolefinas reticuladas ${ }^{[30-31]}$. Os polímeros obtidos têm sido explorados comercialmente como isoladores de cabos elétricos e de dutos para água quente ${ }^{[32,33]}$.

Oemprego de silanos como agentes de acoplamento em compósitos poliméricos vem sendo feito já há algum tempo. Os silanos melhoram significativamente a adesão entre matrizes poliméricas e cargas inorgânicas tais como mica $^{[17]}$, fibra de vidro ${ }^{[34]}$ e talco $^{[35]}$.

Recentemente, Feller e colaboradore ${ }^{[36]}$ descreveram um trabalho em que foi feita a modificação química do PP com silano visando seu emprego como agente de acoplamento para compósitos de PP e fibra de vidro. $\mathrm{O}$ método adotado consistiu na preparação de um copolímero propeno-dieno, que foi submetido a uma hidro-sililação, levando à obtenção de oligopropilenos funcionalizados.

Uma reação de hidro-sililação foi proposta por Sheares ${ }^{[37]}$ para a funcionalização do PP com um hidrosilano. Segundo os autores, na reação entre PP, hidrosilano e peróxido no estado fundido, inicialmente o iniciador promove a quebra da cadeia polimérica, produzindo terminações olefínicas às quais, posteriormente, se adicionam radicais silila, através de um mecanismo de adição radicalar.
O presente artigo descreve um estudo feito sobre a funcionalização de PP isotático com viniltrietoxisilano (VTES) na presença de radicais livres, por meio de reações feitas em solução e em massa.

\section{Procedimento Experimental}

Materiais

Os materiais utilizados foram o polipropileno comercial (PPH301), isento de aditivos, índice de fluidez $12 \mathrm{~g} / 10 \min$ (a 2,16kg), $\bar{M} w=233.000, \bar{M} w / \bar{M} n=6,5$, produzido pela OPP Petroquímica (Triunfo, RS), VTES Silan GF56, produzido pela Wacker, peróxido de dicumila (DCP) $98 \%$ e peróxido de benzoíla (DBP) $70 \%$ da Aldrich Chemical Company.

Os solventes, xileno e acetona comerciais, foram previamente destilados.

\section{Reações em solução}

O PP foi dissolvido em xileno, à temperatura de refluxo, sob atmosfera inerte. Após a dissolução, foi adicionado o VTES e a seguir, uma solução de DBP em xileno. A concentração final de PP no meio foi de $0,04 \%(\mathrm{~m} / \mathrm{V})$. O sistema foi mantido em refluxo durante 3 horas. $\mathrm{O}$ produto foi então precipitado em acetona e seco sob pressão reduzida.

\section{Reações no estado fundido}

Para as reações no estado fundido foi utilizado um misturador Haake Rheomix 600p previamente aquecido a $170{ }^{\circ} \mathrm{C}$ e saturado com argônio. Dois minutos após a introdução do PP na câmara de mistura foi feita a adição de uma solução de DCP em VTES. A velocidade dos rotores foi ajustada para $50 \mathrm{rpm}$. Após o tempo desejado, o produto foi removido, triturado e submetido à pressão reduzida para remoção de compostos voláteis e não reagidos.

\section{Espectrometria no Infravermelho (FTIR)}

Foi utilizado um espectrômetro FTIR Mattson 3020, série Galaxy 3000. As medidas foram feitas utilizando-se filmes preparados a $170{ }^{\circ} \mathrm{C}$, com 2,5 ton $/ \mathrm{cm}^{2}$ de pressão, em uma prensa Carver, série Monarch, modelo 3710-ASTM. O intervalo de números de onda empregado foi de 4000 a $400 \mathrm{~cm}^{-1}$. O grau de funcionalização foi avaliado comparando as bandas de absorção de estiramento assimétrico e simétrico do grupo $\mathrm{Si}-\mathrm{O}-\mathrm{C}$ do polímero funcionalizado 
$\left(1108 \mathrm{~cm}^{-1} \text { e } 1080 \mathrm{~cm}^{-1}, \mathrm{~A} 1\right)^{[38]}$ com a banda de absorção do grupo metila da cadeia de PP $\left(899 \mathrm{~cm}^{-1}\right.$, A2 - referência interna). A razão A1/A2 é proporcional à quantidade de silano incorporada às cadeias.

\section{Cromatografia de Permeação em Gel (GPC)}

Os pesos moleculares foram determinados utilizando-se um cromatógrafo de permeação em gel Waters $150 \mathrm{CV}$ equipado com 3 colunas Styragel HT3, HT4 e HT6 $\left(10^{3}, 10^{4}\right.$ e $\left.10^{6} \AA\right)$ e detector de índice de refração. As análises foram feitas a $140^{\circ} \mathrm{C}$, utilizando-se 1,2,4-triclorobenzeno como solvente. Os pesos moleculares foram calculados usando-se uma curva universal de calibração construída com padrões de polipropileno, polietileno e poliestireno.

\section{Resultados e Discussão}

A Figura 1 mostra os espectros no infravermelho do PP de partida e de um típico PP funcionalizado com VTES, o qual apresenta fortes bandas de absorção a 1080 e $1108 \mathrm{~cm}^{-1}$, correspondentes ao estiramento da ligação

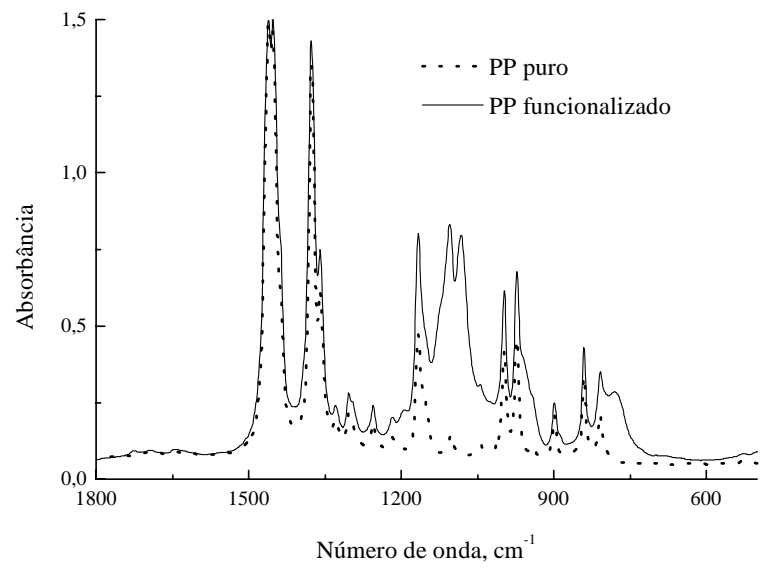

Figura 1. Espectros na região do infravermelho do PP puro e de PP funcionalizado com VTES.

Si-O-C (A1). Essas bandas são relacionadas com a banda de absorção a $899 \mathrm{~cm}^{-1}$ (A2), característica do grupo metila da cadeia polimérica (referência interna). As razões A1/A2 estão diretamente relacionadas à quantidade de VTES incorporada às cadeias, ou seja, ao grau de funcionalização. Métodos matemáticos possibilitam a separação das bandas a 1080 e $1108 \mathrm{~cm}^{-1}$ de outras pequenas bandas referentes ao PP presentes nesta região.

Tabela 1. Reações do PP com VTES em solução

\begin{tabular}{|c|c|c|c|c|c|}
\hline VTES (g\%) & DBP (g\%) & A1/A2 & $\overline{\operatorname{Mn}} \times 10^{-3}(\mathrm{~g} / \mathrm{mol})$ & $\bar{M} w \mathbf{x} 10^{-3}(\mathrm{~g} / \mathrm{mol})$ & $\overline{\mathbf{M}} \mathbf{w} / \overline{\mathbf{M}} \mathbf{n}$ \\
\hline 0 & 0 & 0,6 & 36 & 233 & 6,5 \\
\hline 10 & 1 & 1,0 & 43 & 157 & 3,7 \\
\hline 15 & 1 & 1,0 & 38 & 215 & 5,6 \\
\hline 20 & 1 & 1,1 & 41 & 217 & 5,3 \\
\hline 10 & 2 & 1,0 & 41 & 155 & 3,8 \\
\hline 20 & 2 & 1,1 & 39 & 215 & 5,5 \\
\hline 30 & 2 & 1,1 & - & - & - \\
\hline 60 & 2 & 1,0 & - & - & - \\
\hline 20 & 4 & 1,0 & 44 & 189 & 4,3 \\
\hline 30 & 4 & 1,0 & 40 & 209 & 5,2 \\
\hline 20 & 6 & 1,1 & 39 & 182 & 4,6 \\
\hline 30 & 6 & 1,8 & 44 & 190 & 4,5 \\
\hline 20 & 8 & 2,2 & 51 & 182 & 3,5 \\
\hline 30 & 8 & 1,8 & 32 & 190 & 5,8 \\
\hline 20 & 10 & 1,4 & 42 & 195 & 4,6 \\
\hline 30 & 10 & 1,2 & 50 & 138 & 4,1 \\
\hline
\end{tabular}

Solvente: xileno. Temperatura: $139^{\circ} \mathrm{C}$. Tempo de reação: 3 horas. 
Tabela 2. Reações do PP com VTES no estado fundido

\begin{tabular}{|c|c|c|c|c|c|c|}
\hline VTES (g\%) & DCP $(\mathrm{g} \%)$ & A1/A2 & Torque final (N.m) & $\overline{M n} \times 10^{-3}(\mathrm{~g} / \mathrm{mol})$ & $\bar{M} w \times 10^{-3}(\mathrm{~g} / \mathrm{mol})$ & $\overline{\mathbf{M}} \mathbf{w} / \overline{\mathbf{M}} \mathbf{n}$ \\
\hline $0 *$ & 0,00 & 0,0 & - & 36 & 233 & 6,5 \\
\hline 0 & 0,00 & 0,0 & 2,60 & 48 & 187 & 3,9 \\
\hline 0 & 0,20 & 0,6 & 0,55 & 41 & 85 & 2,1 \\
\hline 1 & 0,10 & 3,3 & 1,00 & 28 & 98 & 3,5 \\
\hline 1 & 0,20 & 4,0 & 0,45 & 30 & 83 & 2,8 \\
\hline 1 & 0,25 & 2,5 & 0,60 & 24 & 87 & 3,5 \\
\hline 1 & 0,30 & 2,8 & 0,20 & 24 & 75 & 3,1 \\
\hline 2 & 0,10 & 2,9 & 1,02 & 28 & 128 & 4,6 \\
\hline 2 & 0,20 & 4,7 & 0,55 & 27 & 89 & 3,3 \\
\hline 2 & 0,25 & 5,1 & 0,35 & 28 & 68 & 2,4 \\
\hline 2 & 0,30 & 2,7 & 0,05 & 28 & 84 & 3,0 \\
\hline 5 & 0,00 & 10,0 & 0,55 & 41 & 136 & 3,3 \\
\hline 5 & 0,10 & 9,3 & 0,70 & 28 & 118 & 4,1 \\
\hline 5 & 0,20 & 10,0 & 0,35 & 24 & 79 & 3,3 \\
\hline 5 & 0,25 & 10,6 & 0,20 & 27 & 96 & 3,5 \\
\hline 5 & 0,30 & 12,1 & 0,30 & 27 & 84 & 3,1 \\
\hline 5 & 0,50 & 16,1 & 0,20 & 22 & 53 & 2,4 \\
\hline 5 & 0,75 & 13,0 & 0,10 & 25 & 51 & 2,0 \\
\hline 5 & 1,00 & 11,1 & 0,00 & 17 & 47 & 2,8 \\
\hline 10 & 0,10 & 18,1 & 0,80 & 44 & 121 & 2,8 \\
\hline 10 & 0,20 & 17,8 & 0,30 & 34 & 82 & 2,4 \\
\hline 10 & 0,30 & 20,5 & 0,15 & 24 & 69 & 2,9 \\
\hline 10 & 0,50 & 23,5 & 0,15 & 25 & 55 & 2,2 \\
\hline 10 & 0,75 & 24,3 & 0,10 & 22 & 69 & 3,1 \\
\hline 10 & 1,00 & 23,7 & 0,00 & 15 & 53 & 3,6 \\
\hline
\end{tabular}

* PP puro, não processado. Temperatura: $170{ }^{\circ} \mathrm{C}$. Tempo de reação: 10 minutos.

Entretanto, a deconvolução não apresentou variação significativa nos resultados obtidos. Os espectros no infravermelho dos polímeros funcionalizados não apresentam absorção na região característica das bandas de carbonila, como se pode verificar na Figura 1, indicando que reações de oxidação não alcançam níveis elevados nestes sistemas.

As Tabelas 1 e 2 mostram a variação do grau de funcionalização e do peso molecular dos produtos obtidos nas reações em solução e no estado fundido, em função das concentrações de silano e peróxido utilizadas. A Tabela 2 mostra, também, o valor do torque final das reações feitas na câmara de mistura.

\section{Grau de funcionalização}

As reações de funcionalização realizadas em solução de xileno foram feitas com concentrações de silano na faixa de $10-30 \%$ e de peróxido de dibenzoíla na faixa de $1-10 \%$ com relação à massa de PP utilizada. Já nas reações conduzidas no estado 
fundido foram usadas concentrações de silano entre 1 e $10 \%$ e de peróxido de dicumila entre 0,10 e $1,00 \%$ com relação à massa de PP. Mesmo assim, como se pode observar nas Tabelas 1 e 2, de acordo com os valores de A1/A2, o grau de funcionalização dos produtos obtidos nas reações no estado fundido foi sempre superior ao dos produtos obtidos nas reações em solução.

A Figura 2 mostra a relação entre a concentração de DBP utilizada no meio reacional e o grau de funcionalização nas reações feitas em solução, para 20 e $30 \%$ de VTES. Pode-se observar que o grau de incorporação de silano é aproximadamente constante no intervalo de concentração de peróxido utilizado, apresentando um aumento entre 6 e $8 \%$ de DBP. Se comparados aos resultados obtidos nas reações no es-

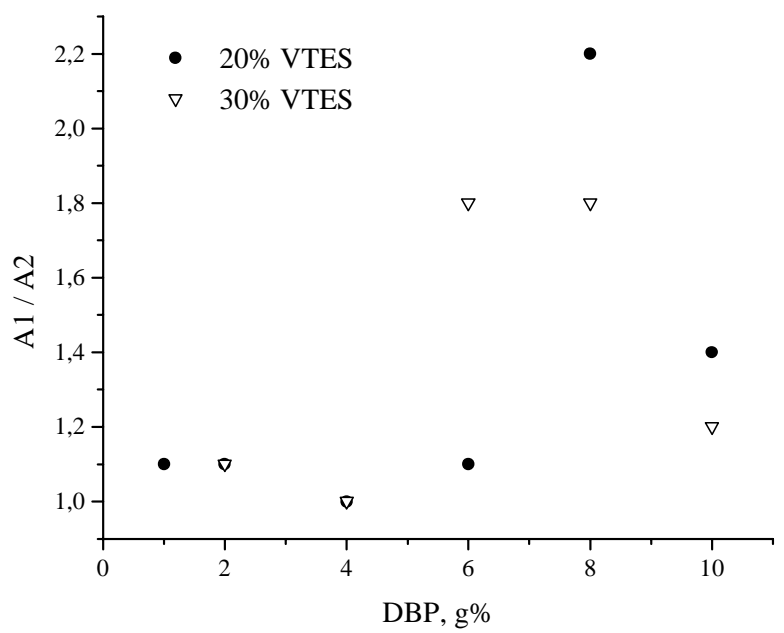

Figura 2. Influência da concentração de iniciador sobre o grau de incorporação de VTES, a $139^{\circ} \mathrm{C}$, em solução de xileno. Tempo de reação: $180 \mathrm{~min}$.

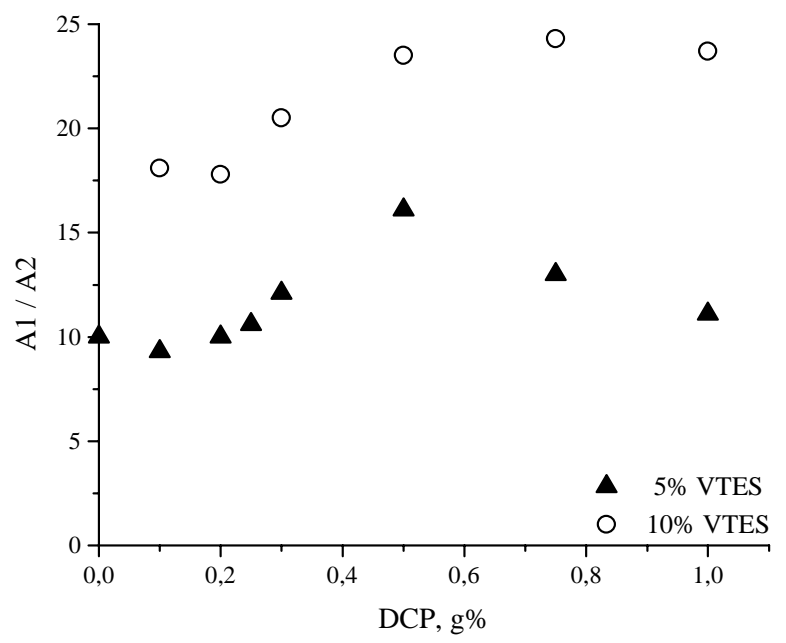

Figura 3. Influência da concentração de iniciador sobre o grau de incorporação de VTES, a $170{ }^{\circ} \mathrm{C}$, nas reações no estado fundido. Tempo de reação: $10 \mathrm{~min}$. tado fundido (Figura 3), verifica-se que esses níveis de incorporação são facilmente ultrapassados quando se usam no misturador concentrações de silano e peróxido a partir de 1 e $0,1 \%$ respectivamente.

O maior nível de incorporação obtido nas reações feitas no estado fundido pode ser explicada por dois diferentes fatores:

a) Maior eficiência dos radicais livres formados a partir do DCP. Esta observação foi anteriormente feita por Minoura e colaboradores ${ }^{[4]}$ ao verificar que, em solução de benzeno, a $120^{\circ} \mathrm{C}$, o peróxido de dicumila apresenta maior eficiência que o peróxido de dibenzoíla nas reações de funcionalização do PP com anidrido maleico, apesar da maior velocidade de decomposição do DBP nessas reações. Os autores explicam a maior eficiência em função da maior estabilidade e polarizabilidade dos radicais livres gerados pelo DCP.

b) Participação do solvente. É provável que o solvente tenha uma participação muito efetiva durante a reação em solução, levando ao consumo de radicais livres do meio reacional. $\mathrm{O}$ solvente utilizado, xileno, pode facilmente perder um átomo de hidrogênio, produzindo radicais benzílicos, de acordo com a Reação 1. Esta reação compete favoravelmente com a reação de formação de macro-radicais terciários a partir do PP (Reação 2), a qual é uma etapa essencial para que ocorra a funcionalização.
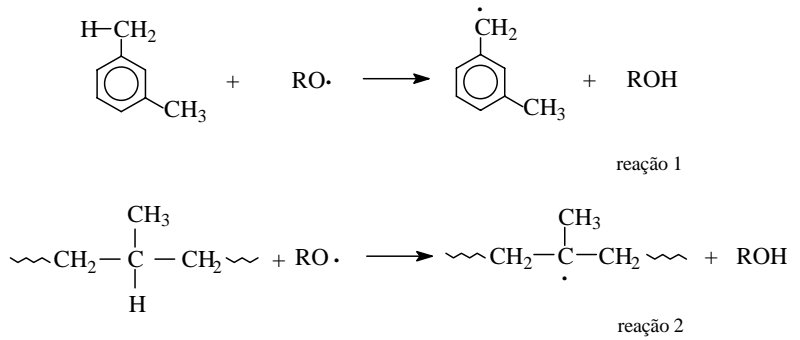

O grau de funcionalização nas reações feitas em solução praticamente não se mostrou dependente das concentrações de silano utilizadas. Até $4 \%$ de peróxido no meio reacional, concentrações de silano de 10 - 60\% resultaram em graus de incorporação semelhantes, conforme os dados da Tabela 1. Entretanto, o grau de funcionalização nas reações feitas no estado fundido aumentou sensivelmente com o aumento na concentração do silano, como se pode verificar pelos dados da Tabela $2 \mathrm{e}$, mais claramente, pela Figura 4. Estas observações sugerem mais uma vez a ocorrência de reações laterais importantes nos 


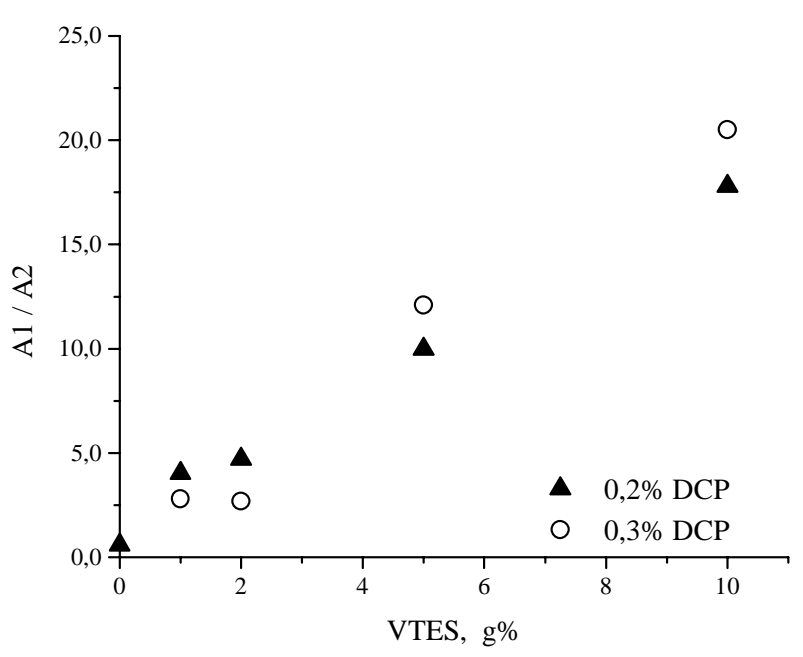

Figura 4. Influência da concentração de silano sobre o grau de incorporação de VTES, a $170{ }^{\circ} \mathrm{C}$, nas reações no estado fundido. Tempo de reação: 10 minutos.

sistemas em solução, as quais promovem o consumo de radicais livres, dificultando a incorporação das moléculas do silano às cadeias poliméricas.

\section{Peso molecular}

Segundo a literatura ${ }^{[7]}$, a reação lateral mais importante na funcionalização radicalar do PP é a quebra de cadeia, através de reações de cisão $\beta$, de acordo com o mecanismo proposto no Esquema 1. Na Figura 5 estão representadas curvas de distribuição de peso molecular típicas de um polímero funcionalizado em solução e de um polímero funcionalizado no estado fundido, além da curva de distribuição de peso molecular do PP de partida. Apesar da alta concentração de radicais livres presentes no meio, as reações feitas em solução não resultaram em uma quebra significativa nas cadeias poliméricas, apontando novamente para um consumo paralelo desses radicais, provavelmente por moléculas do solvente. Por outro lado, as reações feitas no estado fundido, sem a presença de solvente, produziram produtos com menor peso molecular, conforme os dados da Tabela 2 e da Figura 6. Verifica-se que o peso molecular diminui quando a concentração de peróxido aumenta, como resultado do aumento do número de radicais livres presentes, os quais promovem a quebra de cadeia.

A presença de concentrações diferentes de VTES, para as mesmas concentrações de DCP, apresentou pouca influência sobre o peso molecular, como se pode verificar na Figura 6. Analisando-se esta figura e os dados de peso molecular da Tabela 2

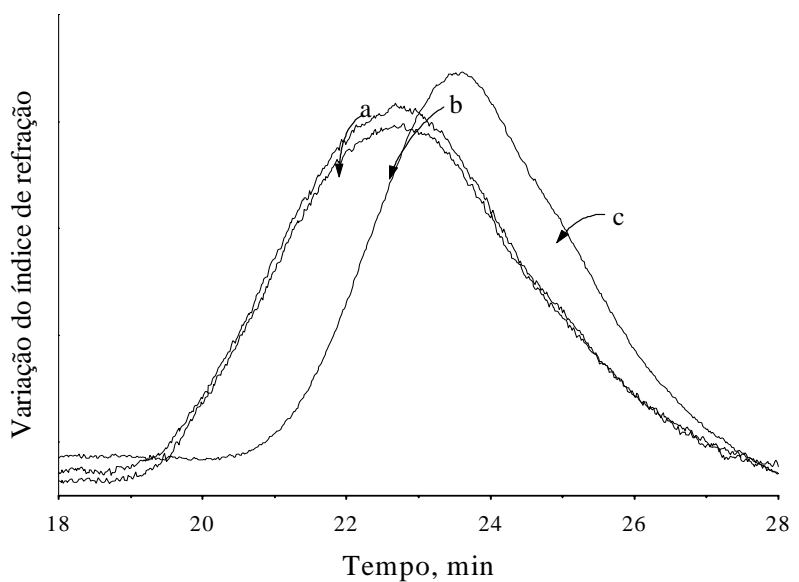

Figura 5. Curvas de distribuição de peso molecular: a) PPH301; b) PP+20\%VTES+8\%DCP (reação em solução); c) PP+2\%VTES+0,3\%DCP (reação no estado fundido).

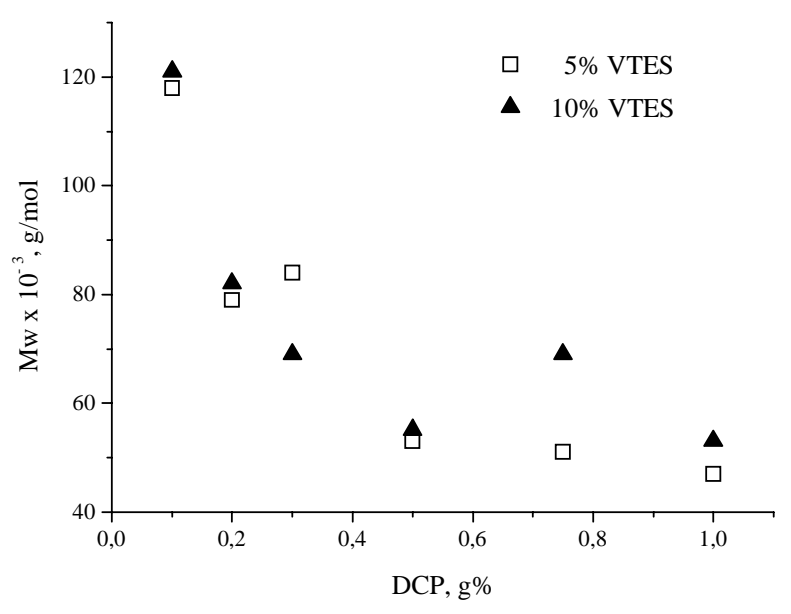

Figura 6. Influência da concentração de peróxido sobre o peso molecular, na funcionalização de PP no estado fundido. Tempo de reação: 10 minutos.

pode-se sugerir que a presença do vinilsilano inibe ligeiramente a extensão das reações de quebra de cadeia.

A Figura 7 apresenta curvas de torque características do PP puro processado nas condições usuais $\left(170^{\circ} \mathrm{C}, 10 \mathrm{~min}, 50 \mathrm{rpm}\right)$ e de dois experimentos típicos. As curvas se caracterizam por um pico inicial resultante da introdução do polímero frio na câmara de mistura, o qual decai após a fusão completa do material, atingindo a seguir um patamar com torque aproximadamente constante. $\mathrm{O}$ valor do torque final está diretamente relacionado com o peso molecular do produto, podendo ser um indicativo ou da ocorrência de quebra de cadeia ou de reticulação. Verifica-se que o torque dos polímeros processados na presença de peróxidos é sempre inferior ao do PP processado sozinho. Quanto maior a concentração de iniciador utilizada, menor é o torque medido, conforme representado na Figura 8. 


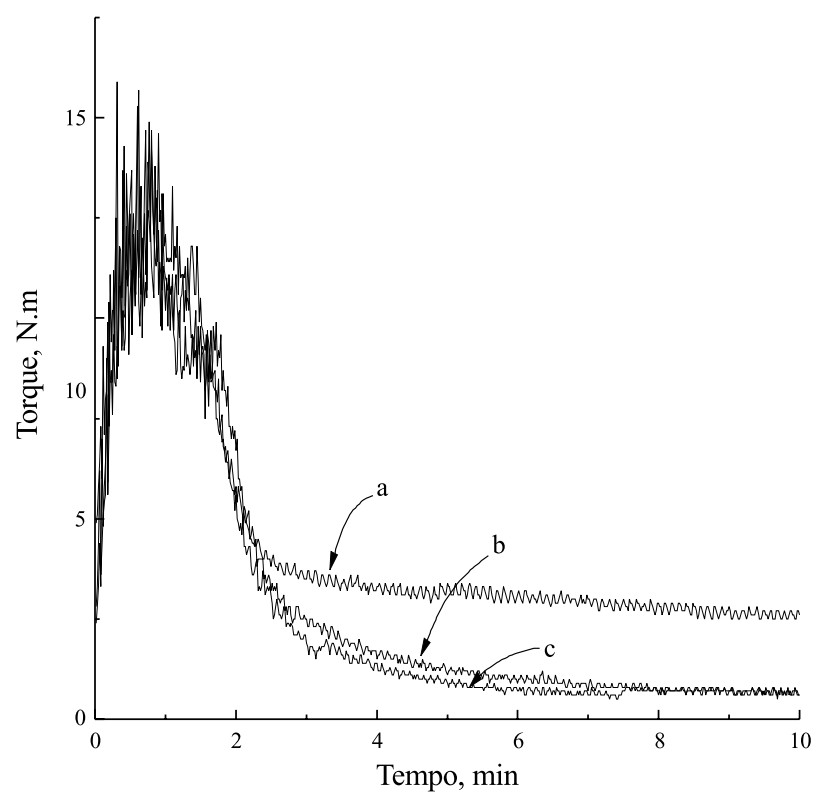

Figura 7. Curvas de torque a $170^{\circ} \mathrm{C}$ : a) $\mathrm{PPH} 301$; b) $\mathrm{PP}+1 \%$ VTES+0,1\%DCP; c) $\mathrm{PP}+5 \% \mathrm{VTES}+0,1 \% \mathrm{DCP}$.

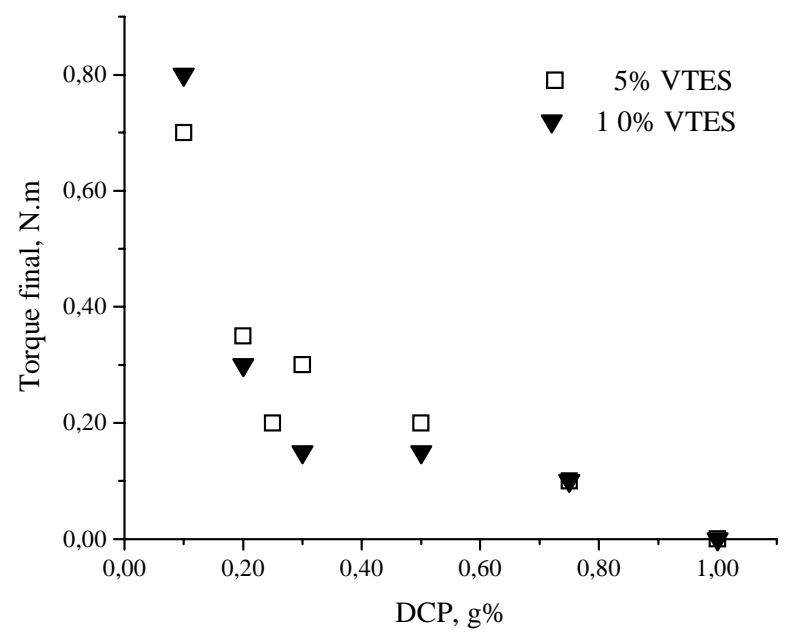

Figura 8. Influência da concentração de DCP sobre o torque final, na funcionalização de PP no estado fundido. Tempo de reação: 10 minutos.

Novamente é possível observar que a variação na concentração de VTES tem pouca influência sobre os valores do torque final. Entretanto, analisando-se a Figura 8 e os dados de torque da Tabela 2, observa-se que o torque mais alto corresponde às menores concentrações de silano, para mesmas concentrações de peróxido. Esta situação é inversa ao que se esperaria em função unicamente dos pesos moleculares (Figura 6) e encontra explicação no fato de que o silano, por ser uma molécula pequena, líquido à temperatura ambiente, exerce um efeito plastificante no meio reacional, diminuindo o torque. Considerando-se essas pequenas variações, é possível concluir que há uma boa concordância entre as medidas de torque e os valores de peso molecular determinados.

\section{Conclusões}

Polipropileno funcionalizado com viniltrietoxisilano foi obtido por meio de reações em solução e no estado fundido, utilizando peróxidos como iniciadores. As reações em solução de xileno apresentaram graus de funcionalização inferiores àqueles obtidos nas reações no estado fundido, mesmo para concentrações muito elevadas de silano e peróxido, da ordem de 20$60 \%$ e $10 \%$ em peso, respectivamente.

A análise dos valores de torque e de peso molecular indica a ocorrência de reações de quebra de cadeia em ambos os processos, mais acentuadamente nas reações feitas no estado fundido. Essas observações indicam que nos sistemas em solução ocorrem reações laterais de consumo de radicais livres e de silano, possivelmente envolvendo moléculas de solvente.

\section{Agradecimentos}

PADCT-NM, CAPES, CNPq, Fapergs, OPP Petroquímica.

\section{Referências Bibliográficas}

1. Guyot, A. - Polym. for Adv. Technol., 7, p.61 (1994).

2. Xu, G. \& Lin, S. - J. Macromol. Sci.- Rev. Macromol. Chem. Phys., C34(4), p.555 (1994).

3. Ide, F. \& Hasegawa, A. - J. Appl. Polym. Sci., 18, p.963 (1969).

4. Minoura,Y.; Ueda,M.; Mizunuma,S. \& Oba, M. - J. Appl. Polym. Sci., 13, p.1625 (1969).

5. Ruggeri, G.; Aglietto, M.; Petragnani, A. \& Ciardelli, F. - Eur. Polym. J., 19(10/11), p.863 (1983).

6. Sathe, S. N.; Rao, G. S. S. \& Devi, S. - J. Appl. Polym. Sci., 53(2), p.239 (1994).

7. Gaylord, N. G. \& Mishra, M. K. - J. Polym. Sci.Polym. Lett. Ed., 21, p.23 (1983).

8. Ho, R. M.; Su, A. C.; Wu, C. H. \& Chen, S. I. Polymer, 34(15), p.3264 (1993). 
9. Liu, N. C.; Xie, H. Q. \& Baker, W. E. - Polymer, 34(22), p.4680 (1993).

10. Rengarajan, R.; Parameswaran, V. R.; Lee, S.; Vicic, M. \& Rinaldi, P. - Polymer, 31(9), p.1703 (1990).

11. Romero, M.; Domard, A. \& Petit, D. - Polymer, 34(14), p.3004 (1993).

12. Citovický, P.; Mikulasová, D.; Chrástová, V.; Mejzlik, J. \& Majer, J. - Die Angew. Chem., 117, p.131 (1983).

13. Lazár, M.; Hrckova, L.; Fiedlerova, A.; Borsig, E.; Rätzsch, M. \& Hesse, A. - Die Angew. Makromol. Chem., 243, p.57 (1996).

14. Markham, R. L. - Adv. Polym. Technol., 10(3), p.231 (1990).

15. Greco, R.; Maglio, G. \& Musto, P. V. - J. Appl. Polym. Sci., 33, p.2513 (1987).

16. Schellenberg, J.; Hamann, B. \& Kaltmasser, H. Angew. Makromol. Chem., 130, p.99 (1985).

17. Chiang, W. Y. \& Yang, W. D. - J. Appl. Polym. Sci., 35(3), p.807 (1988).

18. Rao, M. H. \& Rao, K. N. - Polym. Bull., 1, p.727 (1979).

19. Aglietto, M.; Bertani, R.; Ruggeri, G. \& Ciardelli, F. - Makromol. Chem., 193, p.179 (1992).

20. Aglietto, M.; Ruggeri, G.; Luppichini, E.; D'Alessio, E. \& Benedetti, E. - Materials Eng., 4(2), p.253 (1993).

21. De Vito, G.; Lanzetta, N.; Maglio, G.; Malinconico, M.; Musto, P. \& Palumbo, R. - J. Polym. Sci.: Polym. Chem Ed,, 22, p.1335 (1984).

22. Braun, D. \& Schmitt, M. W. - Polym. Bull., 40, p.189 (1998).
23. De Roover, B.; Sclavons, M.; Carlier, V.; Devaux, J.; Legras, R. \& Momtaz, A. - J. Polym. Sci.: Part A: Polym. Chem., 33, p.829 (1995).

24. Katamura, K. - JP Patent 271619A2 (1994).

25. Kuchiki, J. - JP Patent 112694 (1993).

26. Preston, J. A. \& Davis, C. J. - US Patent 3505279 (1970)

27. Fujimoto, I.; Isshiki, S.; Kurita, Y. \& Sato, Y. - US Patent 4228225 (1980).

28. Daijo, K. \& Kazumi, I. - JP 160852 A2 (1990).

29. Poole, M. J. - U. S. Patent 4136132 (1979).

30. Cartasegna, S. - Rubber Chem. and Technol., 59, p.722 (1986).

31. Sen, A. K.; Mukherjee, B.; Bhattacharyya, A. S.; De, P. P. \& Bhowmick, A. K. - J. Appl. Polym. Sci., 44, p.1153 (1992).

32. Scott, H. G. - US Patent 3646155 (1972).

33. Swarbrick, P.; Green, W. J. \& Maillefer, C. - US Patent 4117195 (1978).

34. Ishida, H. \& Koenig, J. L. - J. Polym. Sci.: Polym. Phys. Ed., 18, p.233 (1980).

35. Han, C. D.; Kwack, T. H. \& Luo, H. L. - J. Appl. Polym. Sci., 29, p.2599 (1984).

36. Feller, J. F.; Chabert, B.; Guyot, A.; Spitz, R.; Wagner, H. D. \& Gerard, J. F. - J. Adhes., 58(3/ 4), p.299 (1996).

37. Shearer, G. \& Tzoganakis, C. - J. Appl. Polym. Sci., 65, p.439 (1997).

38. Bellamy, L. J. - "The infrared spectra of complex molecules”, Chapman and Hall, London, 1975.

Recebido: $27 / 05 / 98$

Aprovado: 09/10/98 